\title{
Adaptation of a Minimal Four-State Cell Model for Reproducing Atrial Excitation Properties
}

\author{
FM Weber ${ }^{1}$, S Lurz ${ }^{1}$, DUJ Keller ${ }^{1}$, DL Weiss ${ }^{1}$, \\ G Seemann ${ }^{1}$, C Lorenz $^{2}$, O Dössel ${ }^{1}$ \\ ${ }^{1}$ Universität Karlsruhe (TH), Karlsruhe, Germany \\ ${ }^{2}$ Philips Research Europe, Hamburg, Germany
}

\begin{abstract}
Simulation of cardiac excitation is often a trade-off between accuracy and speed. A promising minimal, timeefficient cell model with four state variables has recently been presented together with parametrizations for ventricular cell behaviour. In this work, we adapt the model parameters to reproduce atrial excitation properties as given by the Courtemanche model. The action potential shape is considered as well as the restitution of action potential duration and conduction velocity. Simulation times in a single cell and a tissue patch are compared between the two models. We further present the simulation of a sinus beat on the atria in a realistic $3 D$ geometry using the fitted minimal model in a monodomain simulation.
\end{abstract}

\section{Introduction}

When simulating cardiac excitation, one often has to compromise between model accuracy and speed. Detailed but time-consuming ionic cell models reflect reality quite accurately, whereas alternative approaches such as a cellular automaton are faster but suffer from discretization effects. A minimal model (MM) with four state variables has recently been presented [1]. While still using HodgkinHuxley type equations, it performs much better and could be an alternative to such automaton systems. Especially for clinical applications that require patient-specific parameter adaptation, high computational speed is crucial.

The MM calculates the current density of three major currents: a fast inward current $J_{f i}$ (corresponding to $\mathrm{Na}^{+}$), a slow outward current $J_{s o}\left(\mathrm{~K}^{+}\right)$and a slow inward current $J_{s i}\left(\mathrm{Ca}^{2+}\right)$. Current densities are determined by only four state variables $u, v, w$ and $s$, where $u$ is rescaled to the transmembrane voltage (TMV). A set of 28 parameters, mainly time constants and threshold voltages, controls the time evolution of the state variables and thus the shape of the action potential (AP) and other properties such as for example action potential duration (APD) restitution.
While parameter sets reproducing ventricular APs are supplied in [1], there is no setup for atrial excitation yet. In this contribution, we have therefore fitted the model parameters such that it can reproduce atrial APs as generated by the well-known Courtemanche (CM) model [2]. Reproduction of APD as well as conduction velocity $(\mathrm{CV})$ restitution are considered in the adaptation process.

\section{Methods}

The minimal model was implemented into a C++ simulation framework [3]. For optimization, functions depending on the $u$ state variable were tabularized when possible. The implementation was verified against the supplied MatLab example code [1] and showed the correct output for all ventricular parameter sets. The CM model was also available in the framework in a comparably optimized form.

It has to be noted that APs of the CM model differ significantly between simulations in single cells and in tissue patches especially regarding upstroke peak height and APD. For the minimal model, this difference is much smaller. Therefore, reference curves of the CM model at different pacing frequencies were generated using a monodomain finite-difference (FD) simulation in a onedimensional (1D) patch. It consisted of 200 voxels in length with a voxel size of $0.33 \mathrm{~mm}$. The cell was driven at a basic cycle length (BCL) of $1000 \mathrm{~ms}$ for 20 beats after which a transition to a lower BCL (higher frequency) was performed. After another 20 beats, the BCL was decreased again down to a minimum of $260 \mathrm{~ms}$ that could be stimulated without alternans effects to appear. The last beat at each BCL then constituted the reference curve.

APD reference data was generated from these curves. As $\mathrm{APD}_{90}$ is determined by both the upstroke peak height and the falling phase of the AP it is not an optimal criterion for adjusting the AP tail. We therefore selected the point at which the AP reaches $-73 \mathrm{mV}$ as the property to be adjusted (from now on referred to as $\mathrm{APD}_{-73 \mathrm{mV}}$ ). For a BCL of $1000 \mathrm{~ms}$ this only differs to $\mathrm{APD}_{90}$ by a few ms.

Reference data for the conduction velocity were created 


\begin{tabular}{|c|c|c|c|c|c|c|c|c|c|c|c|c|c|}
\hline$u_{o}$ & $u_{u}$ & $\theta_{v}$ & $\theta_{w}$ & $\theta_{v}^{-}$ & $\theta_{o}$ & $\tau_{v 1}^{-}$ & $\overline{\tau_{v 2}^{-}}$ & $\overline{\tau_{v}^{+}}$ & $\overline{\tau_{w 1}^{-}}$ & $\overline{\tau_{w 2}^{-}}$ & $k_{w}^{-}$ & $u_{w}^{-}$ & $\tau_{w}^{+}$ \\
\hline 0 & 1.02 & 0.302 & 0.33 & 0.172 & 0.06 & 65.6 & 1150 & 0.95 & 170.8 & 112.4 & 135 & 0.0744 & 217 \\
\hline$\tau_{f i}$ & $\tau_{o 1}$ & $\tau_{o 2}$ & $\overline{\tau_{s o 1}}$ & $\tau_{\text {so } 2}$ & $k_{\text {so }}$ & $u_{s o}$ & $\overline{\tau_{s 1}}$ & $\tau_{s 2}$ & $\bar{k} k_{s}$ & $u_{s}$ & $\overline{\tau_{s i}}$ & $\tau_{w \infty}$ & $w_{\infty}^{*}$ \\
\hline 0.0678 & 100 & 64.87 & 53.54 & 8.03 & 1.748 & 0.644 & 5.406 & 52.91 & 1.008 & 0.814 & 6.978 & 4.97 & 1 \\
\hline
\end{tabular}

Table 1. Resulting parameter set for reproducing atrial excitation properties with the minimal model.

in the 1D patch described above. First, the tissue conductivity was adjusted to a $\mathrm{CV}$ of $700 \mathrm{~mm} / \mathrm{s}$ at $1 \mathrm{~Hz}$. Next, pacing was started at a BCL of $1000 \mathrm{~ms}$, with the BCL subsequently being decreased down to $260 \mathrm{~ms}$ after 20 beats each. The CV was calculated from the signal delay between two points at $33 \mathrm{~mm}$ distance, centered in the patch.

With the reference data ready, the parameters of the MM are adjusted subsequently. A first rough fit of the AP shape to the reference curve at a BCL of $1000 \mathrm{~ms}$ is performed by manually adjusting $\theta_{w}$ and $\theta_{o}$ and then using the example code from [1]. This invokes a sequential quadratic programming (SQP) method in Matlab. However, the supplied code only fits the initial beat which is not in steadystate. For this beat a BCL cannot be defined either.

The outcome is therefore fed into a Particle Swarm Algorithm (PSA) as a starting point for further optimization in the $\mathrm{C}++$ framework. The PSA search principle is based on the flocking behaviour of for example birds or fish schools $[4,5]$. Movement of a particle in a parameter direction of the search space is influenced by its current velocity (with an inertia weighting factor), the best position it has found so far itself (cognitive factor) and the overall best position found by any of the particles (social factor).

For multi-parameter optimization problems like the present one, a randomized inertia weight factor together with a decreasing cognitive factor and an increasing social factor under the optimization course showed the best results in our tests. A limitation of this setup is that the number of iteration steps must be defined in the beginning in order to adjust the cognitive and social factors. But even with that limitation, it appears to be a favourable method for the optimization of such a multi-dimensional problem that can be assumed to have many local minima.

A first fit is performed for a BCL of $1000 \mathrm{~ms}$ in which the fifth beat of the MM in a single-cell simulation is compared to the reference curve. Due to its relative simplicity, all beat-to-beat variations should be negligible then. The resulting parameter set is further adjusted manually with respect to peak height. $u_{u}$ is tuned until the maximum $V_{m}$ is between 2.0 and $2.5 \mathrm{mV}$, according to the reference value of $2.4 \mathrm{mV}$. The SQP algorithm is further invoked again for a slight adjustment of the spike and dome morphology.

With a good approximation of the AP shape, the PSA fitted a defined set of parameters to match the APD restitution curve to its reference values. Modified were $\tau_{w 1}^{-}$, $\tau_{w 2}^{-}, u_{w}^{-}, k_{w}^{-}$and $w_{\infty}^{*}$ as these are the main contributors to the recovery of the $\mathrm{Ca}^{2+}$ gating variable $w$. Additionally, a small adjustment of $\tau_{s i}$ was allowed.

Finally, the conduction velocity was fitted by adjusting $\tau_{v 1}^{-}, \tau_{f i}$, and $u_{q}$. The velocity of the MM was calculated in an equal simulation setup as the CM reference data. As for each parameter set to be tested a whole patch simulation had to be started, this was most time consuming per parameter evaluation. As already the second beat at each BCL gave a good approximation, its $\mathrm{CV}$ was chosen to be compared to the reference for efficiency reasons.

It should be noted that it is most effective to first adjust the APD parameters and then continue with the $\mathrm{CV}$ tuning. The influence of the APD on CV restitution is rather large. Reactivation of the $\mathrm{Na}^{+}$gates at the rate $\tau_{v 1}^{-}$is voltage triggered. Changes in APD result in delayed or respectively advanced reactivation and thus a change in the upstroke velocity of the next beat for a given BCL. On the other hand, modification of the $\mathrm{CV}$ parameters does not significantly shift the time at which the upstroke peak is reached on the time scale of a whole AP. Thus, APD is affected much less by adjusting the $\mathrm{CV}$ parameters.

A finite element (FE) monodomain (MD) simulation in a tissue patch of $300 \times 300 \times 5$ voxels with a resolution of $0.33 \mathrm{~mm}$ provided a performance test environment in tissue. With a built-in timer, the computational effort for the cell model properties on one hand and the MD excitation

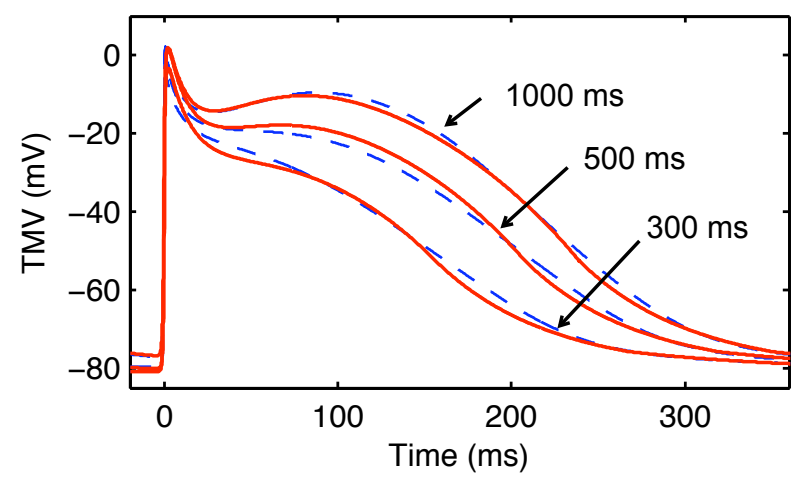

Figure 1. AP shape of the fitted minimal model compared to reference data from CM model. BCLs of $1000 \mathrm{~ms}$, $500 \mathrm{~ms}$ and $300 \mathrm{~ms}$ are shown. The basic shape is well reproduced for all three heart frequencies. 


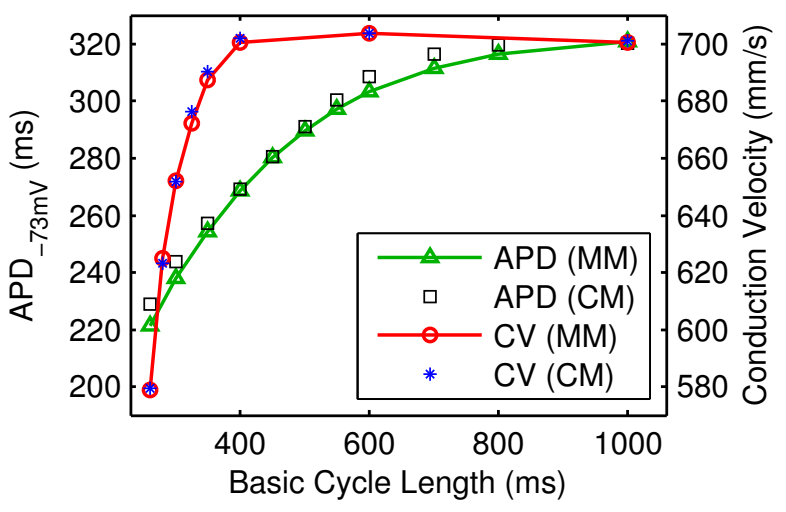

Figure 2. Restitution curves of the fitted MM compared to the $\mathrm{CM}$ reference. For APDs, the time delays are given between the AP peak and the point at which $-73 \mathrm{mV}$ are reached. While single-cell simulations provided the data for the optimization, the APDs shown here were generated in a tissue patch. Despite that, they are in good agreement with the reference data. Further, the $\mathrm{CV}$ restitution curve matches almost perfectly down to $260 \mathrm{~ms}$.

propagation on the other hand could be separated.

While integration time steps of $20 \mu$ s were used in all simulations described so far both for the cell models and the monodomain equation, the MM should tolerate larger steps due to its simplicity. We therefore experimented with increased intervals of $50 \mu$ s to see if the resulting change in CV is well below $5 \%$ as described in [1].

Finally, an isotropic FE monodomain simulation of a sinus beat is performed in a realistic atrial model based on the Visible Female (VF) dataset on two 2,8 GHz QuadCore Intel Xeon CPUs. All cells are pre-paced in singlecell simulations at a frequency of $1 \mathrm{~Hz}$ for 10 beats $(\mathrm{CM})$ or 5 beats (MM) respectively. The simulation is performed for integration time steps of both 20 and $50 \mu \mathrm{s}$.

\section{Results}

The resulting parameter set is shown in table 1 . The rescaling from $u$ to $V_{m}$ in $\mathrm{mV}$ is performed as $V_{m}=85.7$. $u-80.9$. This corresponds to a resting membrane voltage adjusted to $-80.9 \mathrm{mV}$ as observed in the $\mathrm{CM}$ model. Fig. 1 shows the good agreement between the AP curves of the minimal model and the Courtemanche reference.

A quantitative analysis of the APD restitution is shown in fig. 2. The general shape matches well with only slight deviations at BCLs around 300 and 700 ms. It must be emphasized that the demonstrated APDs are calculated in a real tissue patch while the optimization is performed using single-cell simulations. Finally, the dependence of conduction velocity on BCL is calculated as depicted in fig. 2 . It agrees almost perfectly with the reference data.
A first performance test is done in a single-cell simulation where the cell is paced at a rate of $1 \mathrm{~Hz}$ for $10,000 \mathrm{~s}$. On one core of an Intel Core 2 Duo at $2,4 \mathrm{GHz}$, the CM model calculated for $576 \mathrm{~s}$ while the MM with the parameters from table 1 needed only $58 \mathrm{~s}$. This is a time reduction of quite exactly $90 \%$ and demonstrates its efficiency.

On both cores of the same machine we performed a speed test in the previously described tissue patch of $300 \times$ $300 \times 5$ voxels. For simulating $1 \mathrm{~s}$ of excitation, the $\mathrm{CM}$ model required $164 \mathrm{~s}$ while the minimal model finished after $53 \mathrm{~s}$. With a fix-cost amount for the excitation propagation between 30 and $35 \mathrm{~s}$ as well as time for model initialization etc., the remaining calculation time for the cell model was measured to be $111 \mathrm{~s}$ for the $\mathrm{CM}$ model and $12 \mathrm{~s}$ for the minimal model. Again, this is an increase in cell-model calculation speed of more than 9, resulting in an overall speed factor of over 3 .

Simulation time for $1 \mathrm{~s}$ of excitation in the whole atrium using the CM model was around 18.7 hours. The results are shown in fig. 3. However, the minimal model with an integration time step of $20 \mu$ s could not outperform this. With an overall simulation time of $17.5 \mathrm{~h}$ the speed was comparable to the Courtemanche simulation.

When increasing the integration time-steps for the MM to $50 \mu \mathrm{s}, \mathrm{CV}$ decreased by only around $2 \%$ from 702 to $688 \mathrm{~mm} / \mathrm{s}$. This implies the stability of the solution. With tissue conductivity readjusted to a CV of $700 \mathrm{~mm} / \mathrm{s}$, the simulation demonstrated in fig. 3 is performed in $7.8 \mathrm{~h}$ which corresponds to a speed factor around 2.2.

\section{Discussion and conclusions}

We have demonstrated the implementation of a minimal model and adaptation of the parameter set such that it reproduces atrial excitation properties as given by the Courtemanche model. Despite its simplicity, the simulations with the MM show good agreement with the reference data, both for the shape of the AP at different BCLs as well as for the restitution of APD and CV.

The simulated heart beat shows no major differences between the two models (fig.3). The excitation conduction is almost identical. This underlines again the good adaptation of the MM. Only the slightly broadened peak in the MM is manifested spatially in a somewhat elevated TMV behind the depolarization front. The higher integration time steps do not result in increased discretization effects.

Limitations so far are that other properties such as the behaviour under stimulation during the relative refractory period were not considered yet. However, the APD and $\mathrm{CV}$ restitution curves should already approximate the reactivation properties sufficiently well in a first step. Apart from that, iterative repetitions of the procedure could further improve the quality of smaller details of the AP shape (like the peak width) or the APD restitution curve. 

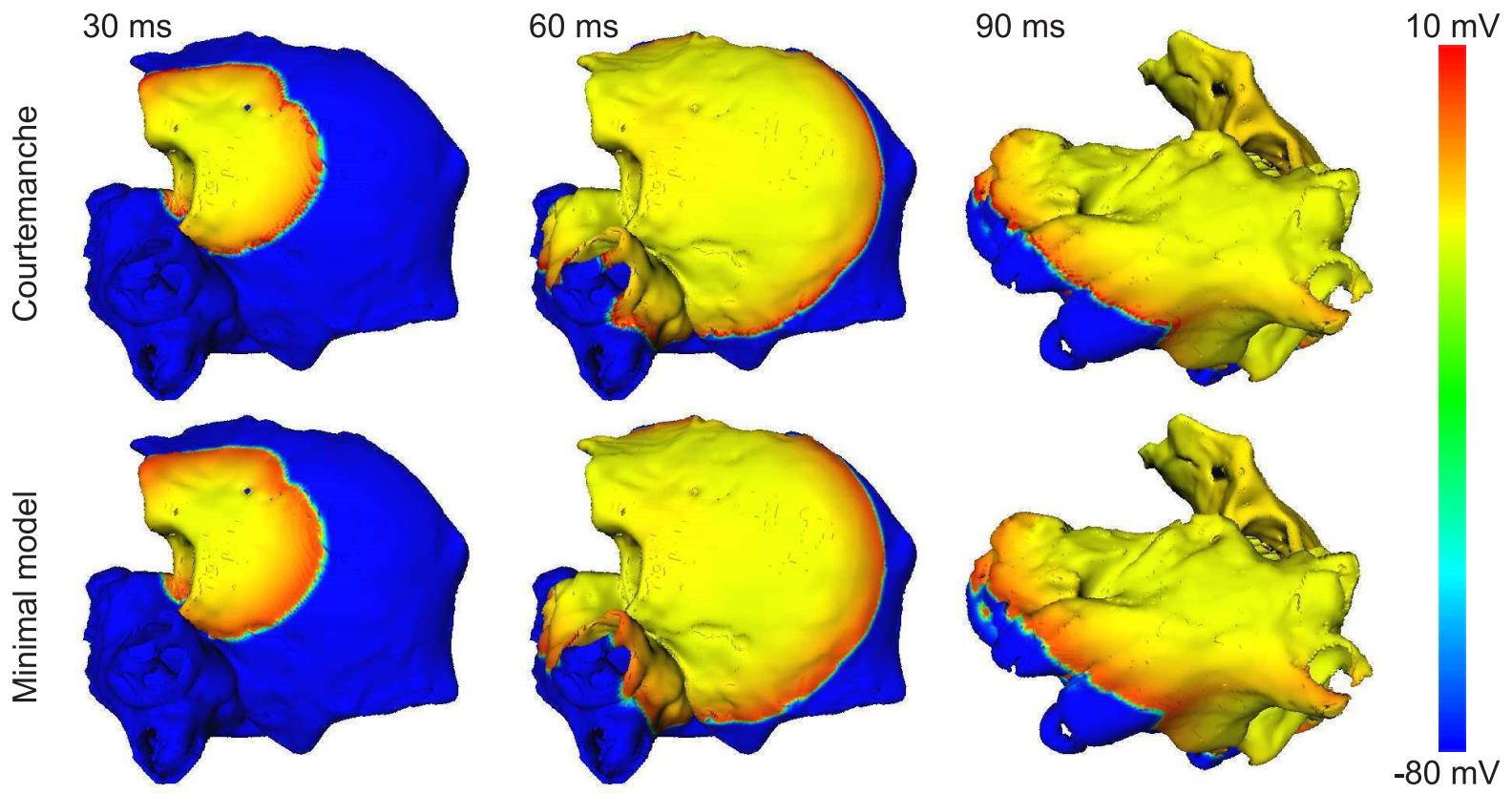

Figure 3. Simulation of a sinus beat on the Visible Female atrial dataset at $t=30,60$ and 90 ms. The transmembrane voltage (TMV) distribution generated with the MM (bottom row) is in good agreement with the CM reference (top row).

We have observed a very good performance of the MM in single-cell and patch simulations. In a large-scale simulation in contrast, the speed-up compared to the CM model vanished for equal iteration steps. However, this does not seem to be a principal restriction of the MM. On one hand, as we found the simulation time being dominated by the MD equation, there is certainly room for improvement. On the other hand, with further adjustments we consider a significant speed-up on the cell model side feasible as well. Apart from that, a first measurable speed factor of more than two is already achieved by the possibility to increase the integration time.

Although the demonstrated properties are well-reproduced, the minimal model cannot account for all the complex behaviour of the full Courtemanche model. While this can be considered a drawback on one hand, it opens up new possibilities on the other hand. Selected features such as APD and CV restitution can be adjusted by a small, defined set of parameters, allowing for controlled variation of these properties. In addition to the speed-up in computation time, this might be an important property when approaching the clinical use of electrophysiological simulations. Firstly, there are still differences to be expected between the simulation in a cell model even of great complexity and a real person's heart. Secondly, clinical use implies the presence of pathologies varying from patient to patient. Thus, model adaptation to patient-specific measurement data is of great importance. A simple, controllable model strongly facilitates such adjustments.
With the presented parameter set, we provide a base for applications of the minimal model regarding atrial physiology and especially pathophysiology, like the study of atrial arrhythmia such as atrial flutter or atrial fibrillation.

\section{References}

[1] Bueno-Orovio A, Cherry EM, Fenton FH. Minimal model for human ventricular action potentials in tissue. Journal of Theoretical Biology 2008;253:544-560.

[2] Courtemanche M, Ramirez R, Nattel S. Ionic mechanisms underlying human atrial action potential properties: Insights from a mathematical model. Am J Physiol 1998;275:301.

[3] Seemann G, Sachse F, Karl M, Weiss D, Heuveline V, Dössel O. Framework for modular, flexible and efficient solving the cardiac bidomain equation using petsc. In Proceedings ECMI. 2008.

[4] Kennedy J, Eberhart RC. Particle swarm optimization. In Proceedings of the IEEE Int. Conference on Neural Networks. 1995; 1942-1948.

[5] Seemann G, Lurz S, Keller D, Weiss D, Scholz E, Dössel O. Adaption of mathematical ion channel models to measured data using the particle swarm optimization. In Proc. EMBEC. 2008.

Address for correspondence:

Dipl.-Phys. Frank M. Weber

Institute of Biomedical Engineering, Universität Karlsruhe (TH)

Kaiserstr. 12, 76128 Karlsruhe, Germany

frank.weber@ibt.uni-karlsruhe.de 